\title{
Curve progression after long-term brace treatment in adolescent idiopathic scoliosis: comparative results between over and under 30 Cobb degrees - SOSORT 2017 award winner
}

\author{
Angelo G. Aulisa ${ }^{1 *}$ (D), Vincenzo Guzzanti ${ }^{1,2}$, Francesco Falciglia ${ }^{1}$, Marco Galli ${ }^{3}$, Paolo Pizzetti ${ }^{1}$ and Lorenzo Aulisa ${ }^{3}$
}

\begin{abstract}
Background: The factors influencing curve behavior following bracing are incompletely understood and there is no agreement if scoliotic curves stop progressing with skeletal maturity. The aim of this study was to evaluate the loss of the scoliotic curve correction in patients treated with bracing during adolescence and to compare patient outcomes of under and over 30 Cobb degrees, 10 years after brace removal.
\end{abstract}

Methods: We reviewed 93 (87 female) of 200 and nine patients with adolescent idiopathic scoliosis (AIS) who were treated with the Lyon or PASB brace at a mean of 15 years (range 10-35). All patients answered a simple questionnaire (including work status, pregnancy, and pain) and underwent clinical and radiological examination. The population was divided into two groups based on Cobb degrees $\left(<30^{\circ}\right.$ and $\left.>30^{\circ}\right)$. Statistical analysis was performed to test the efficacy of our hypothesis.

Results: The patients underwent a long-term follow-up at a mean age of 184.1 months $( \pm 72.60)$ after brace removal. The pre-brace scoliotic mean curve was $32.28^{\circ}\left( \pm 9.4^{\circ}\right)$; after treatment, the mean was $19.35^{\circ}$ and increased to a minimum of $22.12^{\circ}$ in the 10 years following brace removal. However, there was no significant difference in the mean Cobb angle between the end of weaning and long term follow-up period $(p=0.105)$. The curve angle of patients who were treated with a brace from the beginning was reduced by $13^{\circ}$ during the treatment, but the curve size lost $3^{\circ}$ at the follow-up period.

The groups over $30^{\circ}$ showed a pre-brace scoliotic mean curve of $41.15^{\circ}$; at the end of weaning, the mean curve angle was $25.85^{\circ}$ and increased to a mean of $29.73^{\circ}$ at follow-up; instead, the groups measuring $\leq 30^{\circ}$ showed a pre-brace scoliotic mean curve of $25.58^{\circ}$; at the end of weaning, it was reduced to a mean of $14.24^{\circ}$ and it increased to $16.38^{\circ}$ at follow-up.

There was no significant difference in the mean progression of curve magnitude between the $\leq 30^{\circ}$ and $>30^{\circ}$ groups at the long-term follow-up.

Conclusions: Scoliotic curves did not deteriorate beyond their original curve size after bracing in both groups at the 15-year follow-ups. These results are in contrast with the history of this pathology that normally shows a progressive and lowly increment of the curve at skeletal maturity. Bracing is an effective treatment method characterized by positive long-term outcomes, including for patients demonstrating moderate curves.

Keywords: Brace treatment, Adolescent idiopathic scoliosis, P.A.S.B, Long term, Follow-up

\footnotetext{
* Correspondence: angelogabriele.aulisa@fastwebnet.it

'U.O.C. of Orthopedics and Traumatology, Children's Hospital Bambino Gesù,

Institute of Scientific Research, P.zza S. Onofrio 4, 00165 Rome, Italy

Full list of author information is available at the end of the article
} 


\section{Background}

Adolescent idiopathic scoliosis (AIS) is a threedimensional spinal deformity that it is characterized by lateral curvature of the spine and vertebral rotation. The severity of AIS varies greatly, and not all the curves have a progression that requires treatment $[1,2]$.

The most common and conservative approach to treatment of AIS is using a brace to prevent the progression of curvature and in select cases, to obtain a partial recovery of the curve [3-7]. The efficacy of bracing is correlated with longer daily application time and to patient adherence to treatment plans [8-10]. Literature shows the factors that influence curve behavior following bracing are not fully understood, but they are crucial to the prognosis of patients with AIS [10, 11]. Moreover, there is no agreement if scoliotic curves stop progressing after bracing at skeletal maturity.

The aims of this study were:

- To evaluate the loss of the scoliotic curve correction at long term follow-up in a cohort of patients treated with bracing during adolescence;

- To compare the outcomes of sub-group patients: (1) Over and under 30 Cobb degrees at start of treatment, to determine whether the initial curve's gravity could influence long-term results; and (2) over and under 30 Cobb degrees at end of weaning.

\section{Methods}

\section{Patient population}

This is a retrospective study based on an ongoing database including 1512 patients treated for idiopathic scoliosis between 1980 and 2016. Informed consent was obtained by all participants. This study was conducted in accordance with the World Medical Association Declaration of Helsinki of 1975, as revised in 1983, and all the participants signed an informed consent to allow the use of clinical data for research purposes.

A total of 209 scoliotic patients treated with the progressive action short brace (PASB) or Lyon brace were contacted a minimum of 10 years from the end of treatment (range 10-35). Ninety-three patients (87 female) responded to the long-term follow-up examination.

All patients presented at the beginning of treatment, AIS, with curves ranging in magnitude between $20^{\circ}$ and $55^{\circ}$ Cobb. Age at the beginning of treatment was $10-$ 14 years, with Risser scores between 0 and 2 .

\section{Bracing protocol}

All patients were prescribed a full-time (i.e., maximum $22 \mathrm{~h}$ daily, minimum $18 \mathrm{~h}$ daily) brace. For the study, patients showing curve angles $<25^{\circ}$, progression was assessed using two consecutive radiographs taken at 6-month intervals. Progression was defined as an increase greater than $5^{\circ}$ in both curve magnitude (Cobb's method) and apical torsion (Perdriolle's method) in an immature skeleton. Weaning was started when ring-apophysis fusion [12] was seen on a laterolateral view radiograph and consisted of 2 to $4 \mathrm{~h}$ of bracing reduction at 4-month intervals. Short term followup was discontinued 5 years after brace removal. Radiology reports with measurements of the deformity were available for all patients.

\section{Follow up after 10 years since brace removing}

Ninety-three patients were evaluated at long-term follow-up. Demographic characteristics were obtained. Patients were observed in the standing erect position and during the forward bending test.

Full-length anteroposterior (AP) and lateral view standing radiographs were taken. The AP view was used to obtain the curve magnitude (CM, Cobb's method) and torsion of the apical vertebra (TA, Perdriolle's method) $[13,14]$. Measurements were obtained by two independent observers (two senior surgeons) and the end vertebrae were preselected to reduce interobserver bias [14].

All patients answered a simple questionnaire:

- Work Status (yes or no, full or part-time)

- Pregnancy (yes or no, born children)

- Back Pain (yes or no)

\section{Sub group analysis}

The patients were divided in sub-groups based on Cobb degrees. Those with curves $\leq 30$ Cobb degrees and those with curves $>30$ Cobb degrees at the beginning of treatment and at end of treatment.

\section{Statistical analysis}

Standard statistical methods have been used for descriptive statistics. Normally distributed continuous variables were analyzed by using an independent sample $t$ test. Changes in $\mathrm{CM}$ and TA from beginning to follow-up were assessed via one-way analysis of variance for repeated measures. Mean differences between time points and 95\% confidence intervals were calculated. Correlations between changes in CM at the start of bracing, at the end of weaning, and at followup were determined via the Pearson test. All analyses were performed according to the intention-to-treat principle. All tests were two-sided, with significance set at a $P$ value less than 0.05 . Results are presented as mean \pm standard deviation (SD).

\section{Results}

\section{Demographics}

Ninety-three patients (females: 87 ) with a mean age 32.58 $( \pm 5.2)$ years were studied. The mean pre-brace scoliotic curve was 32.28 ( \pm 9.4$)$ degrees, and the mean Perdriolle 


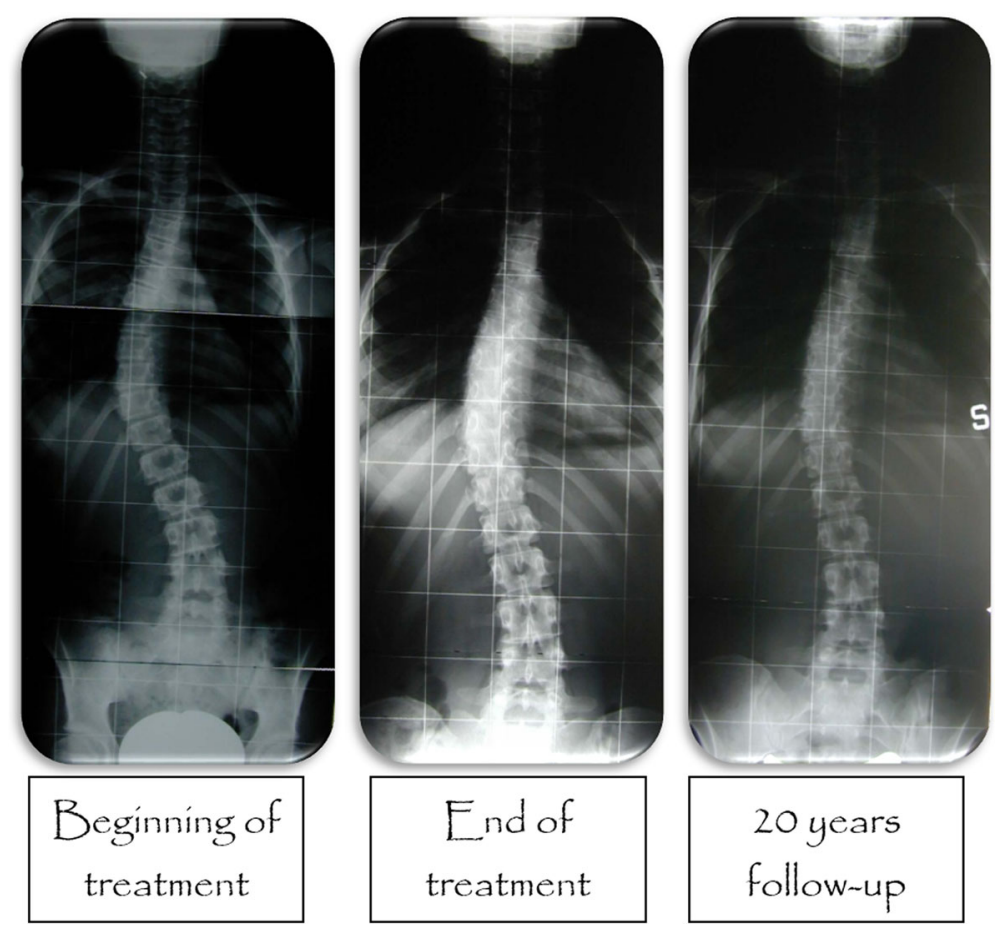

Fig. 1 Typical radiological trend in Cobb degrees of all samples

score of the apical vertebra of the scoliotic deformity was $13.86( \pm 5.04)$.

Twenty-five patients had a thoracic curve, 40 a thoracolumbar or lumbar curve, and 28 had a double primary curve.

Fifty-three patients (57\%) had pre-brace Cobb angle less than $30^{\circ}$, and $40(43 \%)$ had a Cobb angle greater than $30^{\circ}$.

Mean time of brace application was $5.28( \pm 2.23)$ years. Patients underwent long-term follow-up at a mean of $15.33( \pm 5.22)$ years after brace removal.

\section{Cohort results}

In all 93 patients, the mean scoliotic curve was reduced of $13.41^{\circ}( \pm 8.1)$ at the end of weaning.

The mean pre brace Cobb angle of $32.17^{\circ}( \pm 9.12)$ was reduced to $19.39^{\circ}( \pm 10.8)$ following brace removal, it was $20.67^{\circ}( \pm 11.2)$ at the time of the short term follow-up (5 years) and increased to $22.12^{\circ}( \pm 12.11)$ at long-term follow-up. However, there was no significant difference in the mean Cobb angle between the end of weaning and long term follow up period ( $p=0.105)$ (Fig. 1).
Seventy-three patients (78.5\%) completed the long term follow up with less than $30^{\circ} \mathrm{Cobb}$ angle, $11(11.8 \%)$ between 30 and $40^{\circ}$ and nine $(9.7 \%)$ with a Cobb angle greater than $40^{\circ}$. No patients at the long term follow-up demonstrated curve angles greater than $50^{\circ}$.

\section{Comparison of $\leq 30^{\circ}$ vs $>30^{\circ}$ at begging of treatment} Fifty-three patients (57\%) had curve with Cobb degrees $\leq 30^{\circ}$ whereas 40 patients had a curve $(43 \%)>30^{\circ}$.

The group greater than $30^{\circ}$ showed a pre-brace scoliotic mean curve of $41.15^{\circ}$; at the end of weaning, it was reduced to $25.85^{\circ}$ and it increased to $29.73^{\circ}$ at long-term follow-up; instead, the group $<30^{\circ}$ showed a pre-brace scoliotic mean curve of $25.58^{\circ}$; at the end of weaning, it was reduced to $14.24^{\circ}$ and it increased to $16.38^{\circ}$ at long term follow-up (Table 1 and Fig. 2).

Significant differences were determined for CM across Cobb at beginning and at the end of weaning. Instead, insignificant differences were determined for $\mathrm{CM}$ between the end of weaning and the long-term follow-up period (Tables 2, 3).

Table 1 Demographic and radiological characteristics of the study sample

\begin{tabular}{|c|c|c|c|c|}
\hline & Beginning of treatment $\left(t_{1}\right)$ & End of treatment $\left(t_{2}\right)$ & 5 years follow-up $\left(t_{3}\right)$ & 10 years minimum follow-up $\left(t_{4}\right)$ \\
\hline Age (years) & $11.1 \pm 2.4$ & $17.1 \pm 2.0$ & $22.1 \pm 2.5$ & $32.4 \pm 5.1$ \\
\hline Cobb degrees & $32.28 \pm 9.4$ & $19.4 \pm 10.8$ & $20.7 \pm 11.2$ & $22.1 \pm 12.1$ \\
\hline Perdriolle degrees & $13.9 \pm 5.0$ & $9.9 \pm 6.2$ & $10,1 \pm 6.9$ & $10.4 \pm 6.2$ \\
\hline
\end{tabular}




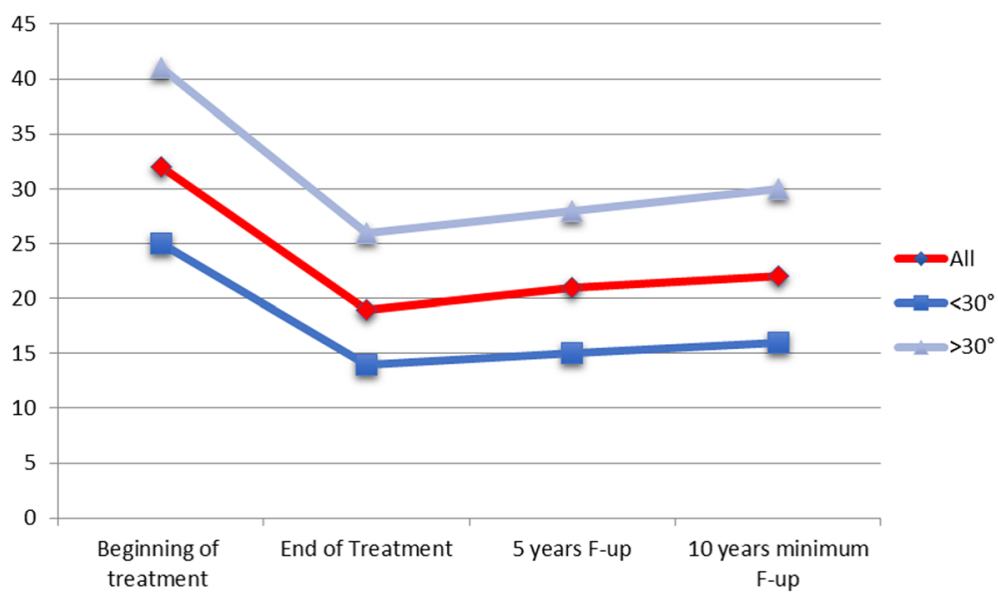

Fig. 2 Radiological trend in Cobb degrees of the all sample and of two groups $<30^{\circ}$ and $>30^{\circ}$

The mean curve correction was $10.94^{\circ}$ in the group with Cobb angles $\leq 30^{\circ}$ and was $15.3^{\circ}$ in the group with Cobb angles $>30^{\circ}$. There was no statistically significant difference in the mean curve correction between the two groups at short term follow-up (Fig. 1).

Long-term follow up revealed a moderate increase in the Cobb angle in both groups. The mean Cobb angle increase was $2.14^{\circ}$ in the group with Cobb angles $\leq 30^{\circ}$ and $3.88^{\circ}$ in the group with Cobb angles $>30^{\circ}$. The difference between groups was not statistically significant $(p=0.87)$.

\section{Comparison of $\leq 30^{\circ}$ vs $>30^{\circ}$ at end of treatment}

Seventy five patients $(81 \%)$ had curve angle $\leq 30^{\circ}$, whereas 18 patients $(19 \%)$ had a curve angle $>30^{\circ}$ at the end of conservative treatment.

The group greater than $30^{\circ}$ showed a pre-brace scoliotic mean curve of $43.94^{\circ}$, at the end of weaning it was reduced to $34.89^{\circ}$ and it increased to $38.39^{\circ}$ at long term follow-up; instead the group $<30^{\circ}$ showed a pre-brace scoliotic mean curve of $29.35^{\circ}$, at the end of weaning it was reduced to $15.05^{\circ}$ and it increased to $18.21^{\circ}$ at long-term follow-up. No significant differences were determined for $\mathrm{CM}$ between end of weaning and long-term follow up period.

Long term follow-up revealed a moderate increase in the Cobb angle in both groups. The mean Cobb angle increase was $3.16^{\circ}$ in the group with Cobb angles $\leq 30^{\circ}$ and $3.50^{\circ}$ in the group with Cobb angles $>30^{\circ}$. Difference between groups was not statistically significant.

\section{Demographics}

Sixty-one patients were employed full-time, 19 were employed part-time, and 13 were unemployed. Twentyone patients experienced pregnancy. Pain, related to instability of the spine, was present in 12 patients ( 3 cases were described as chronic).

\section{Discussion}

The main objective of this study was to evaluate the loss of correction at long term follow-up and to analyze our case series.

Past studies of AIS showed a progression of the curve also at the end of the growth, but the degree of progression was not clear. Weinstein reported that "even in progressive curves it cannot be predicted, for example, whether a progressive $30^{\circ}$ curve's natural history would be to progress to $38^{\circ}$ or to $78^{\circ}$ " [15]. Instead, Bjerkreim [16] reported in his paper about the Progression in Untreated Idiopathic Scoliosis that curve progression was $3^{\circ}$ per year before 20 of age and $1^{\circ}$ per year after 20 years. Curves less than 40 degrees increased significantly less

Table 2 Differences in $C_{M}$ across $t_{1}-t_{4}$ in $<30^{\circ}$ as determined by one-way ANOVA with Bonferroni's post test

\begin{tabular}{|c|c|c|c|c|c|}
\hline $\mathrm{Cobb}<30^{\circ}$ & Mean Diff, & $\mathrm{t}$ & $P<0.05$ & Summary & $95 \% \mathrm{Cl}$ of dif \\
\hline Beginning of treatment $(t 1)$ vs end of treatment ( $t 2)$ & 11.3 & 6.90 & Yes & $* * *$ & 6.96 to 15.7 \\
\hline Beginning of treatment $(t 1)$ vs 5 years follow-up (t3) & 10.5 & 6.49 & Yes & *** & 6.16 to 14.7 \\
\hline Beginning of treatment $(t 1)$ vs 10 years minimum follow-up (t4) & 9.21 & 5.72 & Yes & $* * *$ & 4.92 to 13.5 \\
\hline End of treatment ( $t 2)$ vs 5 years follow-up ( $t 3)$ & $-0,887$ & 0.540 & No & ns & -5.27 to 3.49 \\
\hline End of treatment ( $t 2)$ vs 10 years minimum follow-up ( $t 4)$ & -2.13 & 1.30 & No & ns & -6.51 to 2.25 \\
\hline 5 years follow-up (t3) vs 10 years minimum follow-up (t4) & -1.25 & 0.773 & No & ns & -5.54 to 3.05 \\
\hline
\end{tabular}


Table 3 Differences in $C_{M}$ across $t_{1}-t_{4}$ in $>30^{\circ}$ as determined by one-way ANOVA with Bonferroni's post test

\begin{tabular}{llllll}
\hline Cobb $>30^{\circ}$ & Mean Diff, & t & $P<0.05$ & Summary & $95 \%$ Cl of diff \\
\hline Beginning of treatment $(t 1)$ vs End of treatment $(t 2)$ & 15.3 & 7.36 & Yes & $* * *$ & 9.75 to 20.9 \\
Beginning of treatment $(t 1)$ vs 5 years follow-up $(t 3)$ & 13.2 & 6.37 & Yes & $* * *$ & 7.63 to 18.7 \\
Beginning of treatment $(t 1)$ vs 10 years minimum follow-up $(t 4)$ & 11.4 & 5.53 & Yes & $* * *$ & ns \\
End of treatment $(t 2)$ vs 5 years follow-up $(t 3)$ & -2.15 & 1.04 & No & -7.71 to 3.40 \\
End of treatment $(t 2)$ vs 10 years minimum follow-up (t4) & -3.88 & 1.87 & No & ns & -9.43 to 1.67 \\
5 years follow-up $(t 3)$ vs 10 years minimum follow-up $(t 4)$ & -1.73 & 0.835 & No & ns & -7.24 to 3.79 \\
\hline
\end{tabular}

*** significant

${ }^{\mathrm{ns}}$ not significant

than larger curves and curves measuring from $60^{\circ}$ to $80^{\circ}$ increased the most.

Unfortunately, there are a limited number of studies that support the corrective effect of bracing at the time of the long-term follow-up compared to the natural progression of untreated curves [17-21].

Pellios et al. [17] reported the long term results of 77 patients suffering from AIS at 25 years after Boston brace removal. The initial Cobb angle of $28.2^{\circ}( \pm 8.7)$ was reduced during brace application to $17.3^{\circ}( \pm 9.2)$ then increased at the 25 year follow-up to $25.4^{\circ}( \pm 13.8)$. The mean loss of correction at 25 years after brace discontinuation was $8.1^{\circ}$. Nachemson [20] confirmed similar long-term results of 109 patients, the mean loss of correction at 22 years after brace discontinuation was $7.9^{\circ}$. Lange et al. [21] showed better results in a similar study of 215 patients at 25 years after Boston brace removal. They reported the mean Cobb angle deterioration at the long term evaluation was $4.1^{\circ}$ after brace removal.

In our study, the mean pre-brace Cobb angle of $32.17^{\circ}$ $( \pm 9.12)$ was reduced during brace application to $19.39^{\circ}$ $( \pm 10.8)$. It increased slightly at the short time follow-up to $20.67^{\circ}( \pm 11.2)$ and further increased at the 15-year follow-up to $22.12^{\circ}( \pm 12.11)$. However, the mean prebrace Cobb angle was not significantly different at the 15-year follow-up, demonstrating stability with a loss of correction of $2.7^{\circ}$.

$78.5 \%$ of our cohort completed the long-term follow up with less than $30^{\circ} \mathrm{Cobb}$ angle. Therefore, our results are slightly better than those published in the literature regarding the course of curve progression following brace removal. Bracing seems to be an effective treatment method, with good long-term results also shown in moderate curves.

Furthermore, the results from the subgroups at longterm follow-up revealed a slight increase in the Cobb angle in both groups. The increase was not significantly different at 15 years follow-up and the difference between groups, $2.14^{\circ}\left(\mathrm{Cobb} \leq 30^{\circ}\right)$ versus $3.88^{\circ}\left(\mathrm{Cobb}>30^{\circ}\right)$, was not statistically significant. These results are in contrast with past studies that showed a progressive increment of the curve at skeletal maturity in those that were classified as moderate
$[1,22]$. Reasons effecting the stability of the curve after the brace treatment were not clear from the results but may be related to stiffness of the treated curves.

Using collected demographic data, we found no significant difference in both pregnancy and pain between groups.

A limitation of our study was that the cohort is still young with a mean of 32 years. It would be useful to study patients older than 50 years, in which the degenerative processes of the spine are more evident and important. Even more interesting would be studying the behavior thoracolumbar curves; in fact, the three cases of chronic pain related to instability, due to rotational subluxation, were thoracolumbar curves.

\section{Conclusions}

The results demonstrate slight loss of correction 15 years post bracing. We found no difference in terms of longterm results and progression between patients with $\leq 30^{\circ}$ vs $>30^{\circ} \mathrm{Cobb}$ angles. In conclusion, bracing could be effective for long-term in patients with adolescent idiopathic scoliosis.

\section{Abbreviations}

AIS: Adolescent idiopathic scoliosis; AP: Anterior-posterior; CM: Cobb's method; LL: Latero-lateral; PASB: Progressive action short brace; SOSORT: Society on Scoliosis Orthopedic and Rehabilitation Treatment; SRS: Scoliosis Research Society; TA: Perdriolle's method

\section{Acknowledgements \\ None.}

Funding

No funding was received.

Availability of data and materials

The data sets used and/or analyzed during the current study are available from the corresponding author on reasonable request.

\section{Authors' contributions}

AGA participated in the conception, design, coordination, acquisition, , analysis and interpretation of data, drafting of the manuscript, and performed the statistical analysis. PP, FF, MG, and VG helped to draft the manuscript. LA participated in the conception, design, coordination, and helped to draft the manuscript. All authors read and approved the final manuscript. 


\section{Ethics approval and consent to participate}

Study was conducted with respect to the Helsinki Declaration; all the participants (parents) signed an informed consent to allow the use of clinical data for research purpose.

The Ethics Committee at the Ospedale Pediatrico Bambino Gesù confirmed that retrospective archive studies do not need ethics approval.

\section{Consent for publication}

Not Applicable.

\section{Competing interests}

The authors declare that they have no competing interests.

\section{Publisher's Note}

Springer Nature remains neutral with regard to jurisdictional claims in published maps and institutional affiliations.

\section{Author details}

${ }^{1}$ U.O.C. of Orthopedics and Traumatology, Children's Hospital Bambino Gesù, Institute of Scientific Research, P.zza S. Onofrio 4, 00165 Rome, Italy. ${ }^{2}$ University of Cassino, 03043 Cassino, FR, Italy. ${ }^{3}$ Department of Orthopedics, University Hospital "Agostino Gemelli", Catholic University of the Sacred Heart School of Medicine, 00168 Rome, Italy.

Received: 5 July 2017 Accepted: 18 October 2017

Published online: 30 October 2017

\section{References}

1. Weinstein SL, Ponseti IV. Curve progression in idiopathic scoliosis. J Bone Joint Surg Am. 1983;65(4):447-55.

2. Negrini S, Aulisa AG, Aulisa L, Circo AB, de Mauroy JC, Durmala J, et al. 2011 SOSORT guidelines: orthopaedic and rehabilitation treatment of idiopathic scoliosis during growth. Scoliosis. 2012;7(1):3.

3. Aulisa AG, Guzzanti V, Falciglia F, Giordano M, Marzetti E, Aulisa L. Lyon bracing in adolescent females with thoracic idiopathic scoliosis: a prospective study based on SRS and SOSORT criteria. BMC Musculoskelet Disord. 2015;16:316.

4. Negrini S, Marchini G, Tessadri F. Brace technology thematic series-the Sforzesco and Sibilla braces, and the SPoRT (symmetric, patient oriented, rigid, three-dimensional, active) concept. Scoliosis. 2011;6:8.

5. de Mauroy JC, Lecante C, Barral F, Pourret S. Prospective study and new concepts based on scoliosis detorsion of the first 225 early in-brace radiological results with the new Lyon brace: ARTbrace. Scoliosis. 2014;9:19.

6. Aulisa AG, Guzzanti V, Marzetti E, Giordano M, Falciglia F, Aulisa L. Brace treatment in juvenile idiopathic scoliosis: a prospective study in accordance with the SRS criteria for bracing studies_SOSORT award 2013 winner. Scoliosis. 2014;9:3.

7. Weinstein SL, Dolan LA, Wright JG, Dobbs MB. Effects of bracing in adolescents with idiopathic scoliosis. N Engl J Med. 2013;369(16):1512-21.

8. Aulisa AG, Giordano M, Falciglia F, Marzetti E, Poscia A, Guzzanti V. Correlation between compliance and brace treatment in juvenile and adolescent idiopathic scoliosis: SOSORT 2014 award winner. Scoliosis. 2014;9:6

9. Weiss H-R, Goodall D. The treatment of adolescent idiopathic scoliosis (AIS) according to present evidence. A systematic review. Eur J Phys Rehabil Med. 2008;44(2):177-93.

10. Donzelli S, Zaina F, Negrini S. In defense of adolescents: they really do use braces for the hours prescribed, if good help is provided. Results from a prospective everyday clinic cohort using thermobrace. Scoliosis. 2012;7(1):12.

11. Zaina F, De Mauroy JC, Grivas T, Hresko MT, Kotwizki T, Maruyama T, et al. Bracing for scoliosis in 2014: state of the art. Eur J Phys Rehabil Med. 2014;50(1):93-110.

12. Bick EM, Copel JW. The ring apophysis of the human vertebra. J Bone Jt Surg Am. 1951;33(3):783-7.

13. Omeroğlu H, Ozekin $\mathrm{O}$, Biçimoğlu A. Measurement of vertebral rotation in idiopathic scoliosis using the Perdriolle torsionmeter: a clinical study on intraobserver and interobserver error. Eur Spine J. 1996;5(3):167-71.

14. Morrissy RT, Goldsmith GS, Hall EC, Kehl D, Cowie GH. Measurement of the Cobb angle on radiographs of patients who have scoliosis. Evaluation of intrinsic error. J Bone Joint Surg Am. 1990;72(3):320-7.

15. Weinstein SL. Natural history. Spine. 1999;24(24):2592-600.

16. Bjerkreim I, Hassan I. Progression in untreated idiopathic scoliosis after end of growth. Acta Orthop Scand. 1982;53(6):897-900.
17. Pellios S, Kenanidis E, Potoupnis M, Tsiridis E, Sayegh FE, Kirkos J, et al. Curve progression 25 years after bracing for adolescent idiopathic scoliosis: long term comparative results between two matched groups of 18 versus 23 hours daily bracing. Scoliosis Spinal Disord. 2016;11:3.

18. Wiley JW, Thomson JD, Mitchell TM, Smith BG, Banta JV. Effectiveness of the boston brace in treatment of large curves in adolescent idiopathic scoliosis. Spine. 2000;25(18):2326-32.

19. Maruyama T. Bracing adolescent idiopathic scoliosis: a systematic review of the literature of effective conservative treatment looking for end results 5 years after weaning. Disabil Rehabil. 2008;30(10):786-91.

20. Danielsson AJ, Nachemson AL. Radiologic findings and curve progression 22 years after treatment for adolescent idiopathic scoliosis: comparison of brace and surgical treatment with matching control group of straight individuals. Spine. 2001;26(5):516-25.

21. Lange JE, Steen H, Gunderson R, Brox J.. Long-term results after Boston brace treatment in late-onset juvenile and adolescent idiopathic scoliosis. Scoliosis. 2011:6:18.

22. Tan K-J, Moe MM, Vaithinathan R, Wong H-K. Curve progression in idiopathic scoliosis: follow-up study to skeletal maturity. Spine. 2009; 34(7):697-700.

\section{Submit your next manuscript to BioMed Central and we will help you at every step:}

- We accept pre-submission inquiries

- Our selector tool helps you to find the most relevant journal

- We provide round the clock customer support

- Convenient online submission

- Thorough peer review

- Inclusion in PubMed and all major indexing services

- Maximum visibility for your research

Submit your manuscript at www.biomedcentral.com/submit
) Biomed Central 